\title{
Role of nonlocal exchange correlation in activated adsorption
}

\author{
Hammer, Bjørk; Jacobsen, Karsten Wedel; Nørskov, Jens Kehlet
}

Published in:

Physical Review Letters

Link to article, DOI:

10.1103/PhysRevLett.70.3971

Publication date:

1993

Document Version

Publisher's PDF, also known as Version of record

Link back to DTU Orbit

Citation (APA):

Hammer, B., Jacobsen, K. W., \& Nørskov, J. K. (1993). Role of nonlocal exchange correlation in activated adsorption. Physical Review Letters, 70(25), 3971-3974. https://doi.org/10.1103/PhysRevLett.70.3971

\section{General rights}

Copyright and moral rights for the publications made accessible in the public portal are retained by the authors and/or other copyright owners and it is a condition of accessing publications that users recognise and abide by the legal requirements associated with these rights.

- Users may download and print one copy of any publication from the public portal for the purpose of private study or research.

- You may not further distribute the material or use it for any profit-making activity or commercial gain

- You may freely distribute the URL identifying the publication in the public portal

If you believe that this document breaches copyright please contact us providing details, and we will remove access to the work immediately and investigate your claim. 


\title{
Role of Nonlocal Exchange Correlation in Activated Adsorption
}

\author{
B. Hammer, K. W. Jacobsen, and J. K. Nørskov \\ Physics Department, Technical University of Denmark, DK-2800 Lyngby, Denmark
}

(Received 12 February 1993)

\begin{abstract}
The barrier for dissociative adsorption of $\mathrm{H}_{2}$ on $\mathrm{Al}(110)$ has been calculated within the generalized gradient approximation. A pronounced increase of the barrier height is found compared with what is calculated in the local density approximation (LDA). The apparent LDA underestimation of the barrier height is shown to be intimately linked with the LDA underbinding of core electrons and we suggest it to be a general phenomenon not limited to the particular nonlocal exchange-correlation approximation used or the particular system studied.
\end{abstract}

PACS numbers: 73.20.At, 68.45.Da, 71.10.+x, 82.65.My

The determination of activation barrier heights for adsorption and surface reactions is a subject of considerable interest in modern surface science [1]. Achieving insight into simple systems that can be compared directly with single crystal experiments is the challenge of current theoretical investigations. But the ultimate goal is to be able to predict activation barriers in complex surface reactions of importance in a fundamental description of heterogeneous catalysis.

Density functional theory (DFT) and the local density approximation (LDA) have been extremely successful in predicting adsorption geometries, vibrational properties, and surface diffusion barriers in simple adsorption systems [2-4]. The LDA has also been used to calculate barriers for activated adsorption, but it still remains to be explored how well it works. Comparisons of calculations for $\mathrm{H}_{2}$ dissociation on $\mathrm{Cu}(111)$ [5] and $\mathrm{Al}(110)$ [4] with molecular beam experiments $[6,7]$ indicate that the LDA barriers are too low.

In this Letter we present the first adsorption study within DFT that goes beyond the LDA using a nonlocal exchange-correlation functional - the generalized gradient approximated (GGA) functional of Perdew and Wang [8]. The generalized gradient correction has been shown in a number of studies to consistently improve binding energies of atoms and molecules $[8,9]$. For solid systems the LDA is known generally to overbind and the dominating effect of the GGA is to weaken the binding leading to improved cohesive energies [10]. However, in some cases, like $\mathrm{Pd}$ where the LDA overbinding is absent, the GGA overcorrects with too small cohesive energies and bulk moduli as a result [11].

We study the dissociation of $\mathrm{H}_{2}$ on $\mathrm{Al}(110)$ and find that inclusion of nonlocal exchange-correlation effects in the GGA does not change the properties of the free molecule or the atomically adsorbed state, but drastically increases the barrier for dissociation. The increase is comparable in size to the LDA barrier height and puts into question the validity of the LDA in activation barrier studies. From a detailed analysis of the different contributions to the total energy we conclude that the effect of nonlocal exchange and correlation can be traced to the energy involved in orthogonalizing the metal surface electron states to the adsorbate states. The LDA underestimate of the barrier height turns out to be intimately linked with the well-known LDA failure of underbinding deeply bound electrons $[12,13]$. For this reason, the increase of the dissociation barrier due to nonlocal exchange-correlation effects, reported in this Letter, is suggested to be a general phenomenon not limited to a particular system or nonlocal exchange-correlation energy functional.

The conclusions are based on a set of total-energy calculations for $\mathrm{H}_{2}$ dissociatively adsorbing on $\mathrm{Al}(110)$. The calculations have been performed using a pseudopotential based total-energy method [14]. A complete set of LDA calculations of energy along the lowest energy dissociation path has been reported in Ref. [4]. Here a supercell geometry with a $1 \times 2$ surface cell has been used. The supercell has a five layer $\mathrm{Al}(110)$ slab and five layers of vacuum, and hydrogen is present on both sides of the slab. In the present Letter a similar set of calculations has been performed using the generalized gradient approximation for the nonlocal exchange-correlation functional. We use the GGA version that consists of the refined Becke $[8,15]$ exchange functional and the PerdewWang-91 correlation functional [8]. Compared with the LDA, this GGA version has been found generally to improve the description of total energies, ionization energies, and electron affinities of atoms, atomization energies of molecules, and some solid state properties [8]. Highly nonlocal exchange and correlation effects like the image potential and van der Waals interactions far outside a metallic surface are not included in such a description. As our LDA functional we use the local part of the GGA functional, which is a standard parametrization of the Ceperley-Alder results [16].

The $a b$ initio nonlocal pseudopotential used for aluminum was generated within the LDA. As the adsorption properties of the surface are not expected to depend on the $\mathrm{Al}$ core levels which are most affected by the nonlocal exchange-correlation effects, we use this potential for the GGA study too. The hydrogen atoms are represented by the bare Coulomb potential and all nonlocal effects 
are therefore included here. The one-electron states are expanded in plane waves with kinetic energies up to a cutoff of $500 \mathrm{eV}$. Tests done within both the LDA and the GGA with respect to convergence in basis set, fast Fourier transform grid size, and $k$-point sampling suggest that the activation barrier heights are converged to 0.02 $\mathrm{eV} /$ molecule.

We now turn to the dissociation barrier. In Fig. 1 we compare the LDA and the GGA results for the potential energy per molecule along the reaction path of the dissociative adsorption of $\mathrm{H}_{2}$ on $\mathrm{Al}(110)$. Both curves represent self-consistent calculations where not only the electronic degrees of freedom have been iterated to selfconsistency within either the LDA or the GGA, but where also the steepest descents in ionic coordinates from the saddle points of the LDA and the GGA potential energy surfaces have been done within either approximation. The details of the reaction path are described in Ref. [4].

In accordance with the results calculated by Perdew et al. [8] we find that GGA improves the description of atomic hydrogen and the free $\mathrm{H}_{2}$ molecule over what is found in LDA. However, the change in the atomic chemisorption energy when going from LDA to GGA is not very large as can be seen in Fig. 1. The properties of a chemisorbed hydrogen atom are described very well in both LDA and GGA. For example, the vibrational frequency perpendicular to the surface is found to be 220 $\mathrm{meV}$ in LDA and $225 \mathrm{meV}$ in GGA, both in good agreement with the experimental value [17] of $224 \mathrm{meV}$.

We observe that the dissociation barrier changes dramatically when going beyond the LDA. The LDA barrier is $0.25 \mathrm{eV}$ per molecule, whereas the GGA barrier is $0.54 \mathrm{eV}$ per molecule [18]. This change is in accordance with expectations from a comparison of calculated sticking probabilities from LDA calculated potentials [4] and molecular beam experiments [7].

Despite the large change in the barrier height the minimum energy paths are very similar for the two exchange-

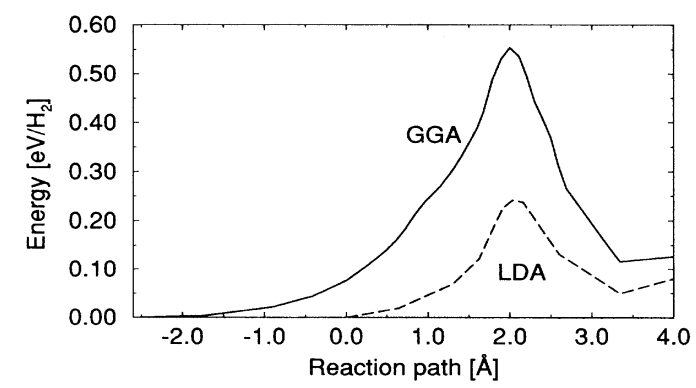

FIG. 1. The LDA (dashed) and GGA (solid) based total energy per $\mathrm{H}_{2}$ molecule dissociating on an $\mathrm{Al}(110)$ surface. The energy is shown along the minimum energy paths determined in the LDA and GGA studies. The reaction coordinate is the length traversed in the six-dimensional space of the molecule [4]. correlation functionals. At the transition state, the $\mathrm{H}-\mathrm{H}$ separation, $b$, and the height of the molecular center of mass, $Z$, above the first $\mathrm{Al}$ ion layer, take the values $(b, Z)=(1.37 \AA, 1.12 \AA)$ and $(1.23 \AA, 1.09 \AA)$, respectively. Indeed, using the LDA ionic coordinates of the initial and transition state in self-consistent GGA calculations gives a dissociation barrier of $0.53 \mathrm{eV}$-only about $10 \mathrm{meV}$ from the GGA barrier of the GGA ionic coordinates.

The barrier height in the GGA can be calculated in a non-self-consistent calculation where the LDA charge density is used. This is a result of the variational properties of the functionals: The error in the energy is second order in the density difference $n^{\mathrm{GGA}}-n^{\mathrm{LDA}}$ which is small. To first order in $n^{\mathrm{GGA}}-n^{\mathrm{LDA}}$ we therefore have

$$
E^{\mathrm{GGA}}=E^{\mathrm{LDA}}+E_{\mathrm{xc}}^{\mathrm{GGA}}\left[n^{\mathrm{LDA}}\right]-E_{\mathrm{xc}}^{\mathrm{LDA}} \text {. }
$$

This means that to first order the change in the barrier height can be calculated directly from the change in the exchange-correlation functional $E_{\mathrm{xc}}$ for a fixed (LDA) density. This procedure leads to an estimate of the dissociation barrier of $0.52 \mathrm{eV}$, very close to the one calculated self-consistently.

The main question at this point is whether the large influence of nonlocal exchange correlation on the barrier height is limited to the GGA and the particular system studied or one can expect similar large effects for other (even better) nonlocal exchange-correlation energy functionals and other activated chemisorption systems. This question cannot be answered simply using the analysis above because it is not clear a priori how large a change another exchange-correlation energy functional would give.

Instead we propose another way of looking at the change in interaction energy between a molecule and a metal surface which directly focuses on the basic reason for the increased barrier and shows that it must be expected to come out from any improvement of the LDA. We will show that the increased repulsion between the molecule and the surface due to nonlocal exchange correlation can be interpreted as arising from an increased repulsion due to the orthogonalization of the metal states to the adsorbate states - the Pauli repulsion. We first demonstrate that the orthogonalization effect increases as we go beyond LDA and afterwards we discuss how this leads to an increased dissociation barrier.

A strong sign of the orthogonalization is observed in the charge density contour plots in Fig. 2. The figure shows the induced densities which we define as the total self-consistent LDA densities with the density of the clean aluminum slab and the density of a free hydrogen molecule with the appropriate nuclear separation subtracted. There is clearly a region around the molecule from which charge has been removed. The orthogonalization of the metal states at the surface to the bonding $\mathrm{H}_{2}$ state requires nodes to be introduced in the metal states 




FIG. 2. (a) The induced LDA charge density when the $\mathrm{H}_{2}$ molecule is positioned parallel to the $\mathrm{Al}(110)$ surface at a distance of $3 \AA$ outside the surface. The plane of the figure is perpendicular to the (110) surface intersecting the $\mathrm{H}$ atoms (small filled circles) and the $\mathrm{Al}$ atoms (large filled circles) forming the long bridge. The induced charge density is obtained by subtracting the density of the clean surface and a free molecule with the same $\mathrm{H}-\mathrm{H}$ bond distance from the total charge density. Thin solid (dashed) lines are contours of positive (negative) charge with a spacing of 0.0005 electron per $\AA^{3}$. The orthogonalization hole at the position of the molecule is clearly seen. (b) Induced LDA charge density with the $\mathrm{H}_{2}$ molecule at the transition state. Again positive (negative) contour lines are solid (dashed), but the spacing between the contours is now 0.005 electron per $\AA^{3}$. The antibonding state of the molecule is being filled by charge transfer from the surface and the orthogonalization hole is restricted to the region between the $\mathrm{H}$ atoms where the antibonding state has a node.

and reduces the weight of these in the region of nonvanishing overlap between the $\mathrm{H}_{2}$ and the surface. Far outside the surface the orthogonalization leads to a shift of electron density from the molecule towards the surface [Fig. 2(a)]. Closer to the surface, at the transition state, the antibonding state of the molecule is being filled by a charge transfer from the surface to the molecule $[19,20]$ [Fig. 2(b)]. The depletion of electron density is therefore limited to the region between the hydrogen atoms where the antibonding state has a node.

The Pauli repulsion is stronger the more localized the adsorbate state is and since the LDA always underbinds core states any improvement of the LDA will give more localized states and thus a stronger orthogonalization. This is also the case with GGA as can be seen in Fig. 3 . In this figure the induced charge densities obtained from self-consistent LDA and GGA calculations are plotted

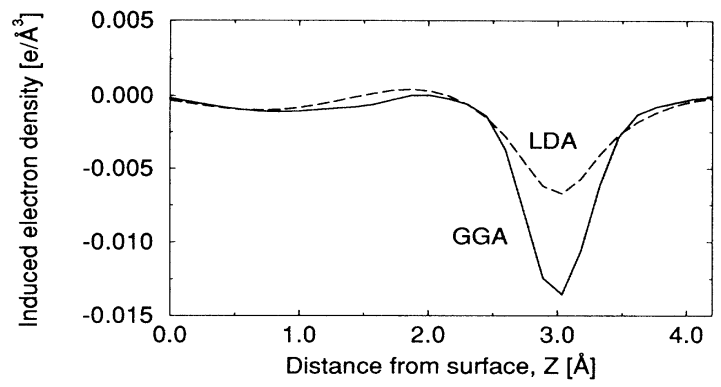

FIG. 3. Induced charge density in LDA and GGA along a line perpendicular to the surface through the center of the molecule. The geometry is as in Fig. 2(a). The orthogonalization hole at the position of the molecule is deepened in GGA compared to LDA.

along a line perpendicular to the surface passing through the center of the molecule. The molecule is positioned parallel to the surface at a distance of $3 \AA$ from the surface [as in Fig. 2(a)]. The orthogonalization hole is clearly deepened in GGA compared with LDA due to the more tightly bound molecular state. It should be noted that the electron density from the clean metal surface does not change significantly as we go from LDA to GGA in accordance with the general picture that LDA describes the valence states reasonably well.

In order to establish the connection between the deepening of the orthogonalization hole and the increased dissociation barrier we consider the asymptotic behavior where the molecule is far away from the surface. In this regime the increased repulsion is also clearly seen in GGA (Fig. 1). Following Zaremba and Kohn [21] and Harris and Liebsch [22] the interaction energy $\Delta E$ between the molecule and the surface can to first order in the overlap between the metal and the molecule be written as [23]

$$
\Delta E=\sum_{k, \text { occ }} \Delta \epsilon_{k}
$$

where the sum is over the energies of the continuum states of the system. Equation (2) can be derived in the context of density functional theory using the force theorem [24]: By shifting the density and potential rigidly along with the atom the contributions to the energy change from electrostatics, and from the intra-atomic part of the exchange and correlation are to lowest order canceled by the counter term in the kinetic energy. The total energy change is therefore given by the change in the eigenvalue sum alone [Eq. (2)] if highly nonlocal interatomic contributions to exchange and correlation (i.e., image effects) are neglected.

The change in the eigenvalue sum, Eq. (2), has been analyzed by Harris and Liebsch [22] in the form of an expansion in the pseudopotential of the adsorbate. They conclude that for adsorbates with deep lying levels, like the noble gases and $\mathrm{H}_{2}$, the kinetic energy cost caused by the orthogonalization of the metal states to the adsorbate level is larger than the potential energy gain. The result 
is a repulsive interaction between the adsorbate and the substrate. This must also be the origin of the repulsion between $\mathrm{H}_{2}$ and the $\mathrm{Al}(110)$ surface seen in Fig. 1. An increase in the orthogonalization energy therefore leads to a stronger repulsion between the substrate and the adsorbate.

We therefore suggest that any nonlocal exchangecorrelation description that corrects for the LDA underbinding of deeply lying states will increase the repulsion between the $\mathrm{H}_{2}$ molecule and the surface. Further, any adsorption system where a barrier for adsorption and dissociation is dominated by orthogonalization effects are expected to show the same behavior.

The orthogonalization effects which dominate the behavior in the asymptotic region far from the surface are also important at the transition state as evidenced by the charge density plot in Fig. 2(b). The situation is more complex here because there is also a beginning filling of the antibonding $\mathrm{H}_{2}$ states which signifies the start of the breaking of the $\mathrm{H}-\mathrm{H}$ bond and the formation of $\mathrm{H}$-surface bonds [19]. This means that the asymptotic expansion cannot be expected to work here, but still the basic physics associated with the orthogonalization hole will be important and dominate the difference between the LDA and nonlocal exchange-correlation treatments.

In conclusion we have presented the first investigation of the effect of including nonlocal exchange-correlation terms in a density functional determination of a dissociation barrier. We have shown that for $\mathrm{H}_{2}$ dissociating on $\mathrm{Al}(110)$, the barrier increases from 0.25 to $0.54 \mathrm{eV}$ when going from the LDA to the GGA. This trend is in accordance with expectations from a comparison of calculated sticking probabilities from LDA calculated potentials $[4,5]$ and molecular beam experiments $[6,7]$. We have pointed out that the increase of the dissociation barrier can be understood in terms of an increase of the orthogonalization energy of the surface states being penetrated by an adsorbate state that has been more tightly bound by the nonlocal exchange-correlation terms. The picture applies independently of the particular nonlocal exchange-correlation functional as this should in general correct for the LDA underbinding of core electrons in atoms.

We appreciate discussions with David Bird. We are grateful to Michael C. Payne for giving us the CASTEP (Cambridge Serial Total Energy Package) code and to J. Perdew for providing the GGA-II code. The research was supported by the Danish research councils through the Danish Center for Surface Reactivity.

[1] For recent experimental and theoretical reviews, see
B. E. Hayden, in Dynamics of Gas-Surface Interactions, edited by C. T. Rettner and M. N. R. Ashfold (The Royal Society of Chemistry, London, 1991), p. 137; J. K. Nørskov, Rep. Prog. Phys. 53, 1253 (1990).

[2] P. J. Feibelman and D. R. Hamann, J. Vac. Sci. Technol. A 5, 424 (1987).

[3] P. J. Feibelman, Phys. Rev. Lett. 67, 461 (1991).

[4] B. Hammer, K. W. Jacobsen, and J. K. Nørskov, Phys. Rev. Lett. 69, 1971 (1992).

[5] J. Müller, Surf. Sci. 272, 45 (1992).

[6] C. T. Rettner, D. J. Auerbach, and H. A. Michelsen, Phys. Rev. Lett. 68, 1164 (1992).

[7] H. F. Berger and K. D. Rendulic, Surf. Sci. 253, 325 (1991).

[8] J. P. Perdew, J. A. Chevary, S. H. Vosko, K. A. Jackson, M. R. Pederson, D. J. Singh, and C. Fiolhais, Phys. Rev. B 46, 6671 (1992).

[9] G. Ortiz and P. Ballone, Phys. Rev. B 43, 6376 (1991).

[10] X. J. Kong, C. T. Chan, K. M. Ho, and Y. Y. Ye, Phys. Rev. B 42, 9357 (1990); G. Ortiz, Phys. Rev. B 45, 11328 (1992); M. Körling and J. Häglund, Phys. Rev. B 45, 13293 (1992).

[11] A. Garcia, C. Elsässer, J. Zhu, S. G. Louie, and M. L. Cohen, Phys. Rev. B 46, 9829 (1992).

[12] R. O. Jones and O. Gunnarsson, Rev. Mod. Phys. 61, $689(1989)$

[13] J. P. Perdew, in Electronic Density Functional Theory of Molecules, Clusters, and Solids, edited by D. E. Ellis (Kluwer, Dordrecht, 1993).

[14] M. C. Payne, M. P. Teter, D. C. Allan, T. A. Arias, and J. D. Joannopoulos, Rev. Mod. Phys. 64, 1045 (1992).

[15] A. D. Becke, Phys. Rev. A 38, 3098 (1988).

[16] D. M. Ceperley and B. J. Alder, Phys. Rev. Lett 45, 566 (1980).

[17] H. Kondoh, M. Hara, K. Domen, and H. Nozoye, Surf. Sci. 268, L287 (1992).

[18] We note that for the present supercell the closest distance between $\mathrm{H}_{2}$ molecules in neighboring cells is $2.8 \AA$. If a monoclinic cell with a minimum distance of $4.9 \AA$ is used instead the barrier heights increase by $0.16 \mathrm{eV}$ for both LDA and GGA. The presence of the intermolecular interactions in the small unit cell does not therefore affect the differences between LDA and GGA.

[19] J. K. Nørskov, A. Houmøller, P. Johansson, and B. I. Lundqvist, Phys. Rev. Lett. 46, 257 (1981).

[20] D. Bird (unpublished).

[21] E. Zaremba and W. Kohn, Phys. Rev. B 15, 1769 (1977).

[22] J. Harris and A. Liebsch, J. Phys. C 15, 2275 (1982).

[23] In the Hartree-Fock based derivation by Harris and Liebsch [22] the van der Waals interaction is taken into account as an additional term.

[24] A. R. Mackintosh and O. K. Andersen, in Electrons at the Fermi Surface, edited by M. Springford (Cambridge Univ. Press, Cambridge, England, 1980); K. W. Jacobsen, J. K. Nørskov, and M. J. Puska, Phys. Rev. B 35, 7423 (1987). 